knowledge, no similar study has been performed in a multiethnic Asian population. This will allow identification of gaps in our healthcare system that can be addressed to decrease the proportion of inappropriate PED attendances. In the COVID19 era, we also aim to review how these trends have changed in a pandemic.

Methods A retrospective study was performed on all attendances to the largest PED in Singapore from 1 January to 31 July 2019 and 2020. A total of 153,631 visits were included for analysis.

Attendances were classified into appropriate or inappropriate depending on resources used and eventual disposition. An appropriate attendance (AA) was defined as fulfilling any of the following criteria: (1) investigated in emergency department (excluding urine studies in children aged 3 years and older); (2) treated in emergency department (excluding basic enteral anti-pyretic medications, prescription medications, or performing of simple procedures); or (3) admitted to the inpatient ward or discharged with specialist follow-up. All other attendances were hence classified as inappropriate attendances (IAs).

Univariate and multivariate analysis was performed to identify predictors of inappropriate attendances.

Results A total of 31,657 attendances (20.6\%) were classified as inappropriate attendances. On multivariate analysis, the three most significant factors predicting inappropriate attendances were lower triage acuity (P3 vs P1, OR 37.37, 95\%CI 27.73-50.36), mode of arrival (self vs ambulance or police escort, OR 1.76, 95\%CI 1.60-1.94), and first visits (re-attendance within 72 hours vs first visit, OR 0.56 , 95\%CI 0.50 0.64). Attendances in 2020 decreased by $40.2 \%$ from 2019, with a smaller proportion of inappropriate attendances in 2020 (21.7\% in 2019 vs $18.8 \%$ in 2020 , p < 0.001). Similarly, abscondment rates $(0.41 \%$ in 2019 vs $0.31 \%$ in 2020$)$ and re-attendance rates $(1.4 \%$ in 2019 and $1.2 \%$ in 2020) decreased in 2020.

Conclusions This study identified the frequency of inappropriate visits, and predictors of these visits. From our data, possible initiatives to decrease inappropriate PED use would be to equip PCPs with facilities for basic fingerpick blood tests for the paediatric population. This study paves the way for direction of future research and educational efforts towards these groups to decrease inappropriate use of the emergency department.

\section{INTERGENERATIONAL PERSPECTIVES OF PATERNAL PARENTING PRACTICES: A DESCRIPTIVE QUALITATIVE STUDY}

Hui Ee Chang, Shefaly Shorey, Qian Wen Sng. Singapore

\subsection{6/bmjpo-2021-RCPCH.183}

Background Parenting practices are behaviours that are known to be challenging and complex. Differences in these behaviours have been proven to have significant implications in child's health and development. Research shows how paternal parenting behaviours can mould the concept of fatherhood for the next generation.Female participation in the workforce, dual income families are on the rise. This promotes father's involvement in child care. Despite the increment in father's participation, the perspectives of fathers, especially grandfathers, remains under-explored. There is a need to further

\begin{tabular}{ll} 
Abstract 333 Table 1 & \\
\hline $\begin{array}{l}\text { Personal traits influencing father's } \\
\text { perspectives }\end{array}$ & Moral value \\
& Personal Beliefs \\
External influences that shape & Societal views about \\
parenting & fatherhood \\
& Cultural influences \\
& Observations in real life \\
Personal views on parenting & Individual parenting \\
& experiences \\
& Past childhood \\
& experiences \\
& Exposure and knowledge \\
& about parenting \\
Child's traits that influence parenting & Situational Circumstances \\
& Child's demographics \\
Child's personality,
\end{tabular}

understand the relationship between a father and a son and how it shapes fatherhood. No such study has been conducted in a multicultural context like Singapore.

Objectives The study aims to explore the intergenerational perspectives of paternal parenting practices in Singapore.

Methods A descriptive qualitative approach was adopted. Purposive sampling was used to recruit 12 dyads (grandfathers and fathers) from the tertiary public pediatric hospital in Singapore. Face-to-face interview with a semi-structured guide was conducted. Thematic analysis was employed for data analysis.

Results Four themes and 13 sub themes related to the influences that affect grandfathers' and fathers' perspectives on parenting were generated. The four main themes are shown in table 1 below:

Conclusions The study explored grandfathers' and fathers' views, beliefs and experiences on paternal parenting. Findings provided us the information to compare between two generations, deepening the understanding of a father-son's relationship, while adding valuable insights to the topic of fatherhood. Findings also encourage improvements to policy and practice, that are important for fathers to assimilate into fatherhood and promote positive child outcomes.

\section{IRON AND VITAMIN D DEFICIENCY IN YOUNG CHILDREN WITH AUTISM SPECTRUM DISORDER IN SINGAPORE}

Magdalena Yvonne Koh, Aishworiya Ramkumar. Singapore

\subsection{6/bmjpo-2021-RCPCH.184}

Background Iron and vitamin D have been implicated to play an important role in cognition and neurodevelopment respectively. Existing evidence suggests that correcting deficiencies in both these nutrients can improve Autism Spectrum Disorder (ASD) symptoms. Children with ASD are also at greater risk for these deficiencies due to behavioural difficulties and food selectivity. Extant literature shows highly variable rates of these deficiencies and variable practices in routine screening among different populations. 
Objectives The objectives of this study were to 1 . Determine occurrence of iron and vitamin D deficiency in the sample population, 2. Identify predictors of the presence of these deficiencies if any and 3. Elucidate factors influencing screening for these deficiencies in children with ASD.

Methods This was a retrospective cross-sectional review of case records of all patients with ASD who were seen at a tertiary developmental paediatric centre in Singapore from January 2018 to June 2018. Inclusion criteria was 1. Child age 0 to 7 years and 2. Diagnosis of ASD following clinical evaluation by a developmental paediatrician or formal psychological evaluation with the Autism Diagnostic Observation Schedule. Exclusion criteria was 1. Chronic medical conditions and 2. Genetic syndromes. Information on demographic variables, ASD-related variables and other medical conditions was abstracted using a structured data collection form. Presence of iron deficiency (serum ferritin $<12 \mu \mathrm{g} / \mathrm{L}$ or transferrin saturations $<10 \%)$, vitamin D deficiency $(25$-hydroxyvitamin D $[25$ $(\mathrm{OH}) \mathrm{D}]<10 \mu \mathrm{g} / \mathrm{L})$ and vitamin $\mathrm{D}$ insufficiency $(25(\mathrm{OH}) \mathrm{D}$ between 10.1-29.9 $\mu \mathrm{g} / \mathrm{L}$ ) was determined from review of laboratory investigations. Descriptive statistics were used to assess for prevalence of iron and vitamin D deficiencies. Logistic regression was used to identify predictors of iron and vitamin $\mathrm{D}$ deficiencies and chi square tests were used to compare children who were and were not offered screening for these deficiencies.

Results The sample consisted of 480 children (81\% males, $19 \%$ females) with a mean age of 4.5 years (SD 1.3). Of this, only $20.2 \%(\mathrm{~N}=97)$ of children were screened for iron deficiency and $18.3 \%(\mathrm{~N}=88)$ were screened for vitamin $\mathrm{D}$ deficiency using blood tests. The prevalence of iron deficiency was $19 \%(\mathrm{~N}=18)$. Younger children were more likely to have iron deficiency $(\mathrm{B}=1.06, \mathrm{p}=0.02)$. The prevalence of vitamin $D$ insufficiency was $38 \%(\mathrm{~N}=33)$ and that of vitamin $\mathrm{D}$ deficiency was $1.1 \%(\mathrm{~N}=1)$. Older children were more likely to have vitamin $\mathrm{D}$ deficiency or insufficiency $(\mathrm{B}=1.07, \mathrm{p}=0.01)$. Only $20.8 \% \quad(\mathrm{~N}=100)$ of children from the entire sample were offered screening blood tests; children with greater severity of disease $(\chi 2=9.80, \mathrm{p}=0.002)$ and those with a history of selective feeding $(\chi 2=8.27, \mathrm{p}=0.004)$ were more likely to be offered screening tests.

Conclusions The prevalence of both iron deficiency and vitamin D deficiency/insufficiency was high in this sample compared to the general population. Of note, only a small proportion of children were offered screening and eventually screened for these deficiencies. Apart from child age, there were no significant child or disease characteristics that predicted these deficiencies. Routine screening of all children with ASD for iron and vitamin D deficiency is recommended.

\section{FRACTURE BURDEN IN PAEDIATRIC END STAGE KIDNEY DISEASE}

Samuel Chung-sum Ho, Eugene Yu-hin Chan, Ellen Yu, Matthew Hon-lam Lee, Yuetling Tung, Alison Lap-tak Ma. Hong Kong

\subsection{6/bmjpo-2021-RCPCH. 185}

Background Paediatric patients with chronic kidney disease are known to have an increased risk of fracture. However, data pertaining to children with end stage kidney disease (ESKD) receiving renal replacement therapy (RRT) is limited.
Objectives The aim of this study is to determine the incidence of fracture and associated factors in this specific group of patients.

Methods We conducted a retrospective review on all paediatric patients with ESKD at the tertiary Paediatric Nephrology Centre in Hong Kong. Children who presented before 18 years with active follow-ups for 12 or more months by November 2020 were included.

Results RRT was initiated in 69 children $(55 \%$ boys) at a mean age of $9.2 \pm 5.9$ years. At the time of evaluation, 21 (30.4\%), $10(14.5 \%)$ and $38(55.1 \%)$ patients received peritoneal dialysis (PD), haemodialysis (HD) and kidney transplant respectively. 3 patients $(4.3 \%)$ had prior kidney graft loss and resumed on dialysis. One patient (1.5\%) reported a fracture prior to RRT.

Over a median of 5.2 years (IQR 3.0-8.3) follow-up, 10 fracture episodes were observed in 7 patients $(10.1 \%)$ at a mean duration of $7.8 \pm 8$ years since RRT initiation, corresponding to a cumulative fracture incidence of 227.8 per 10000 patient year (95\% CI, 86.6-369.0). This rate was 5folds higher than published data from our local general paediatric population (45 per 10,000 person-years; 95\% CI, 43.9$46.1 ; \mathrm{p}=0.01)$. Of note, all patients experienced single fracture episode except one child who developed 4 fracture episodes.

Children who sustained fractures were significantly younger at the time of RRT initiation ( 3.5 vs 10.4 years; $p=0.02)$ and had a longer time on dialysis (12 vs 2.7 years; $\mathrm{p}<0.001)$. Other factors associated with fractures included metabolic bone disease $(28.6 \%$ versus $1.6 \%$; $=0.03)$, difficulty in walking $(28.6 \%$ versus $3.2 \% ; \mathrm{p}=0.05)$, radiological evidence of renal osteodystrophy $(85.7 \%$ vs $25.8 \% ; \mathrm{p}=0.003)$, parathyroid hyperplasia/adenoma $(100 \%$ vs $31.8 \% ; \mathrm{p}=0.01)$ and a higher parathyroid hormone level (pmol/L) (62.7 vs 30.3; p=0.02). Calcium, phosphate and ALP levels, as well as the proportion of patients with vitamin D deficiency were similar between the two groups. While the practice of native and active vitamin $\mathrm{D}$ and phosphate binders were not different, more patients with fracture received cinacalcet $(57.1 \%$ versus $12.9 \%$; $=0.02)$, which may suggest more severe hyperparathyroidism.

Conclusions Children with ESKD receiving RRT are at a higher risk of fracture. Longer duration of dialysis and a higher average parathyroid hormone level were potential modifiable factors associated with fractures.

\section{INTEGRATED LEARNING THROUGH SIMULATIONS WITH THE AVIATION INDUSTRY}

Kee Wei Phang, Poonam Patel. UK

\subsection{6/bmjpo-2021-RCPCH.186}

Background The aviation industry has been the leader in the field of simulation having pioneered and designed much of their current simulation training including crew resource management, situational awareness and debriefing tools. Increasingly, there is an interest in incorporating key aspects of such simulation training into the medical teaching curriculum as many of the skills, particularly with regards to human factors are transferrable skills to medicine. With this in mind and the given the current COVID pandemic which has rendered much of the aviation industry to a halt, the simulation faculty at 